\title{
Cultura festiva, política local y hegemonía social: Comparativa de los casos de los Moros i Cristians (Alcoi), las Falles (València) y La Patum (Berga) / Festive culture, local politics and social hegemony: comparative cases of Moors and Christians (Alcoi), the Fallas (Valencia) and the Patum (Berga)
}

\author{
Verònica Gisbert Gracia \\ Departamento de Sociología y Antropología Social. Universitat de València. España / Spain \\ veronica.gisbert@uv.es
}

\author{
*Joaquim Rius-Ulldemolins \\ Departamento de Sociología y Antropología Social. Universitat de València. España / Spain \\ joaquim.rius@uv.es
}

\section{Gil-Manuel Hernàndez i Martí \\ Departamento de Sociología y Antropología Social. Universitat de València. España / Spain gil.hernandez@uv.es}

Recibido / Received: 27/11/2017

Aceptado / Accepted: 28/02/2018

\section{RESUMEN}

La cultura festiva se ha convertido, a finales del siglo xxI, en un elemento crecientemente central en la vida social, la identidad territorial y de la economía de las ciudades. A medio camino de la reproducción tradicional, la patrimonialización y la innovación, la cultura festiva ha sido analizada en sus vertientes simbólicas, económicas o turísticas, pero no de forma adecuada como un espacio de disputa de la hegemonía cultural y social en el que la identidad y el orden social tradicional entran en tensión con las iniciativas orientadas a la inclusión social y la igualdad de género. A partir de tres casos muy relevantes por su dimensión local e internacional, los Moros i Cristians (Alcoi), las Falles (València) y la Patum (Berga), se analizará este conflicto entre la esfera tradicional de la cultura festiva y el cambio político, así como las diversas estrategias de gobernanza.

Palabras clave: Festividad, tradición, hegemonía, política cultural, inclusión social, discriminación de género.

\section{ABSTRACT}

The festive culture has become, at the end of the 21st century, an increasingly central element in social life, the territorial identity and in some cases the economy and politics of the cities. Halfway to traditional reproduction, patrimonialization and innovation, the festive culture has been analysed in its symbolic, economic or tourist aspects, but not in an adequate approach as a social space for dispute over cultural and political hegemony in which identity and the traditional social order is in tension with the initiatives aimed at social inclusion and gender equality. From three very relevant cases due to its local and international dimension, Moros i Cristians (Alcoi), Falles (Valencia) and Patum (Berga) the paper analyses this conflict between the traditionalist festive cultural sphere and innovative politics, as well as the various strategies of management of the festivities that lead to a reproduction of the social order and its inequalities or strategies that contribute to greater inclusiveness and equality.

Keywords: Festivity, tradition, hegemony, cultural policy, social inclusion, gender discrimination.

*Autor para correspondencia / Corresponding author: Joaquim Rius-Ulldemolins (Universitat de València). Departament de Sociologia i Antropologia Social. Universitat de València. Facultat de Ciències Socials. Universitat de València. Av. Tarongers, 4b. 46021 València

Sugerencia de cita / Suggested citation: Gisbert Gracia, V., Rius-Ulldemolins, J., Hernàndez i Martí , G. M. (2019). Cultura festiva, política local y hegemonía social: Comparativa de los casos de los Moros i Cristians (Alcoi), las Falles (València) y la Patum (Berga). Revista Española de Sociología, 28 (1), $79-94$. 
Cultura festiva, política local y hegemonía social: Comparativa de los casos de los Moros i Cristians (Alcoi)...

\section{INTRODUCCIÓN: CULTURA FESTIVA, POLÍTICA LOCAL Y HEGEMONÍA SOCIAL}

Desde los años ochenta, las culturas festivas locales se han convertido en un elemento central en la promoción de las ciudades y de su industria turística (Ekinci y Hosany, 2006). En este contexto los festivales y celebraciones tradicionales han ganado una nueva centralidad, especialmente en el sur de Europa (Boissevan, 1992). Este nuevo impulso de las tradiciones ha sido revindicado como la pervivencia de la sociabilidad festiva y como generador de cohesión social (Ariño Villarroya, 1992; X. Costa, 2001). Una sociabilidad que, lejos de oponerse a la modernización, la complementa generando espacios públicos de participación ciudadana (X. Costa, 2002). Además, es ésta la que pone en evidencia que la teoría sociológica de la modernidad ha minusvalorado: la importancia de la tradición y de las identidades territoriales, así como su capacidad de evolucionar e impulsar el cambio (Leoussi, 2013; Shils, 2006). De este modo, las fiestas tradicionales y su innegable vitalidad en las sociedades contemporáneas avanzadas constituyen, sin duda, un desafío a esta visión simplificadora de la modernidad y muestran la pervivencia de formas de sociabilidad basadas en una relectura en constante cambio de la tradición (Ariño Villarroya y Gómez, 2012). Estas dinámicas sociales, además de cohesionar e integrar, en cuanto son singulares de un territorio, le confieren atractivo y sus relecturas generan sensaciones y narrativas que se muestran "auténticas" a los ojos de los actores sociales (instituciones gestoras y organizadoras, ciudadanía participante, turistas, etc). En un mercado global de marcas territoriales, cada vez más uniformizadas, los territorios que han conservado 0 reinventado las tradiciones disponen de un recurso diferenciador que les identifica y singulariza (Ren y Blichfeldt, 2011).

Sin embargo, podemos afirmar que una tradición no es siempre benéfica para una sociedad o para sus miembros, y que esta se caracteriza por una ambivalencia. Así, puede tener consecuencias no sólo funcionales sino también disfuncionales, evitando 0 restringiendo, por ejemplo, la creatividad o la innovación y proporcionando soluciones establecidas de antemano a problemas contemporáneos (Sztompka,
1993). También la tradición puede contribuir a confiar por inercia en las formas de vida, en métodos de gobierno y en estrategias económicas tradicionales en lugar de buscar un cambio en las condiciones sociales. Una inercia o persistencia que puede reflejarse en la forma de concebir las festividades tradicionales en su forma de gestionarse, en sus usos en la conservación de hegemonías políticas basadas en la adscripción. Al mismo tiempo, ello puede favorecer la reproducción de roles de género sexistas y discriminatorios hacia las mujeres o el colectivo LGTB, una dimensión de la que se está tomando mayor conciencia desde hace veinte años a nivel ciudadano y político (Roggeband, 2012). Por ello, hace falta reconocer y analizar la "cara oscura" de la cultura festiva y del capital social que en ella se genera (Putnam, 2002), fenómeno que, en el ámbito cultural, se revela mediante el cierre de grupo, así como en la reproducción de pautas que dificultan la transformación de la cultura en una arena abierta a la participación social inclusiva y diversa (Rius-Ulldemolins et al., 2017). Más allá, por lo tanto, de la necesidad de equilibrio entre tradicionalismo acrítico y anti-tradicionalismo amnésico que plantea Sztompka (1993), es necesario analizar los obstáculos a la modernización de las tradiciones como se refleja en el caso de las festividades tradicionales. Además, el cambio socio-cultural operado en las sociedades avanzadas en general y en particular en España hacia un mayor predominio de las pautas igualitarias en el género y en el retroceso de las creencias religiosas tradicionales, sitúan a las festividades tradicionales en creciente contradicción con estas pautas (Arroyo Menéndez, M., Cabrera Sánchez, J., 2011).

Ciertamente, la política cultural ha jugado un rol esencial en la modernización, la revitalización y la relativa apertura a la participación social en las tradiciones. Un ejemplo destacado es el Estado español, en el que éstas se han convertido en uno de los elementos vertebradores de la vida ciudadana, de la identidad local y de la promoción turística (Hernàndez i Martí, 2016). Esta política ha sido celebrada como una forma de creación de comunidad y de reafirmación de las identidades territoriales (Ariño Villarroya y Gómez, 2012; Ayuntamiento de Zaragoza, 2014). No obstante, desde nuestro punto de vista, la promoción de las fiestas debería ser 
Cultura festiva, política local y hegemonía social: Comparativa de los casos de los Moros i Cristians (Alcoi)...

analizada estudiando, además, su cara oculta como dispositivo de dominación y de reproducción de los mecanismos de desigualdad de acceso a las posiciones de poder, una perspectiva que puede basarse en los conceptos de violencia simbólica de Bourdieu y de hegemonía de Gramsci (Burawoy, 2012; Jeanpierre, 2011). Desde esta perspectiva, la promoción de la cultura puede interpretarse como una herramienta de reproducción de una doxa y un orden social desigual, pero además, como una arena de lucha política por la hegemonía en términos gramscianos, tal y como se ha hecho mayoritariamente desde los estudios culturales (C. Gray, 2010; Merli, 2013).

La relación entre política cultural y hegemonía política en las ciudades ha sido estudiada de forma creciente desde los años ochenta centrando los análisis en sus objetivos, dinámicas y efectos sociales y urbanos (Connolly, 2011; C. Gray, 2002; RiusUlldemolins y Zarlenga, 2014). Sin embargo, menos atención ha recibido la relación entre gobernanza local, hegemonía política y gestión de la cultura tradicional 0 de las fiestas más allá de su relevancia para la reafirmación de la cultura local y la innegable dimensión económica y turística (Cohen, 2013; Dowling, 2008; Gligorijevic, 2014). Entendemos, sin embargo, que las entidades de organización de los eventos festivos tradicionales constituyen, al mismo nivel que las instituciones culturales comúnmente consideradas en relación a la hegemonía como museos o centros culturales (Merli, 2013), dispositivos centrales en el control y la legitimación del calendario social, la identidad local y, en consecuencia, también del orden social (Delgado, 2003).

Desde este punto de vista, en este artículo nos proponemos analizar el combate por la hegemonía en la fiesta y en consecuencia en la política local por parte de diversos sectores con diferentes posiciones en el eje izquierda y derecha y en el eje tradicionalista y anti-tradicionalista ${ }^{1}$. En primer

1 Ciertamente, se puede establecer vínculos entre las tradiciones locales y la identidad regional o nacional, pero dista de ser un vínculo estable y mecánico del que se pueda deducir una función en el marco del desarrollo del nacionalismo y el estado nación. Efectivamente, los elementos culturales y la invención de la tradición juega un rol importante en la identidad nacional (Hobsbawn, 1998), pero no todas las tradiciones juegan el mismo rol. lugar, encontramos la derecha tradicionalista que se erige en defensora de la ortodoxia y de una continuidad inmutable de la fiesta, una posición que normalmente ha gozado de un amplio consenso y que ha convivido con gobiernos también de izquierda, generalmente ajenos al tradicionalismo (íbidem) ${ }^{2}$. La novedad, quizá, es el surgimiento en el Estado español, de una derecha teóricamente anti-tradicionalista y de una izquierda, que sin ser tradicionalista, considera los elementos culturales y simbólicos como una parte importante del combate político por la hegemonía (Sola y Rendueles, 2017). Este giro ha generado un nuevo campo de juego y un nuevo objeto de disputa en la política cultural acerca de la gestión de la fiesta y sus efectos socio-políticos.

En algunos casos las tradiciones locales se han reinventado y extendido en un territorio para ser parte de procesos de nation building, como es el caso de los castellers (Giori, 2012), en otros casos conforman parte de un regionalismo compatible con la adhesión al estado nación como es el caso de las Falles (Ariño Villarroya, 1992; Flor, 2011) 0 en otros casos conforman un discurso de exaltación local, que se encuentra distribuido a lo largo de territorios y regiones distintas, como los Moros y cristianos (Santamarina, 2008). No es objeto de este estudio hacer un análisis en profundidad de esta cuestión, la relación entre tradiciones locales e identidad nacional, que desbordaría el objeto del artículo. Sin embargo, desde el enfoque del que partimos concebimos las identidades locales y nacionales como un espacio de lucha ideológica en la que el estado, las instituciones y una diversidad de agentes del campo político y cultural pugnan para redefinir a partir de elementos culturales precedentes una definición legítima y un control de sus usos discursivos y prácticos (Bourdieu, 1980; Thiesse, 1999).

2 Las posiciones en el campo político se habían estructurado en un eje entre una derecha tradicionalista y una izquierda anti-tradicionalista. Si analizamos los programas políticos, en este caso la derecha era la única a tener una política hacia la fiesta y en los programas de izquierda la fiesta era 0 bien ausente 0 bien ocupaba una posición marginal. La explicación a este "olvido" por parte de la izquierda política tradicional de corte socialdemócrata es que no han querido intervenir en la fiesta, considerándola 0 bien un elemento menor de la agenda política de izquierdas centrada en el estado del bienestar 0 bien un elemento potencialmente peligroso por generador de batallas culturales de tipo identitario en el que muchas veces la izquierda, como es el caso en el País Valenciano ha salido perdiendo (Hernàndez i Martí et al., 2014). 
Los casos de estudio elegidos son los Moros $i$ Cristians de Alcoi (País Valencià), les Falles de València (País Valencià) y la Patum de Berga (Catalunya). Se trata de tres fiestas locales tradicionales de gran importancia y proyección regional e internacional. Las tres han obtenido reconocimientos externos de relevancia; tanto La Patum como las Fallas, fueron declaradas Patrimonio Inmaterial de la Humanidad por la UNESCO en 2005 y 2016 respectivamente.(UNESC0, 2008). Así mismo, los Moros i Cristians son considerados un evento de Interés Turístico Internacional desde 1980. Más allá de su proyección internacional, las tres fiestas constituyen un lugar central en la vida local, no solo durante el periodo festivo, sino también durante el resto del año, pues son componentes esenciales en la construcción de las identidades colectivas de cada población, centrando una gran parte de la atención social y mediática, pero también, política. En los tres casos las festividades ocupan una posición de gran peso en las agendas públicas locales y son objeto de controversia por parte de todos los grupos políticos presentes en el gobierno local, generando, en no pocas ocasiones, debates políticos sobre la reproducción 0 el cambio de la fiesta. Estos debates suscitan controversias entre los grupos de derecha e izquierda, siendo foco importante de estas discusiones: la legitimidad de la tradición, la necesidad del cambio y de abrir la fiesta a una mayor participación ciudadana, tanto en la gestión y el desarrollo festivo, sin excluir a una parte importante de la ciudadanía, como ahora, las mujeres 0 las personas migrantes. Los dilemas se trasladan, de forma a veces conflictiva, a los colectivos organizadores de las festividades, relativamente autónomos, que constituyen organismos centrales en la gobernanza festiva y, más en general, para la hegemonía política y social a través del control de la esfera cultural (Gramsci, 2011). En este sentido, los tres casos seleccionados para el presente artículo, son muy significativos para analizar la intensa relación entre festividad, tradición y hegemonía social y política, que es el objeto de análisis del presente texto ${ }^{3}$.

3 El artículo parte del trabajo de investigación de los autores en relación a la cultura festiva, la gobernanza local,

\section{CULTURA FESTIVA, PATRIMONIO CULTURAL Y POLÍTICA CULTURAL}

En los últimos decenios, coincidiendo con la aceleración del proceso de modernización y de cambio social en Europa, en general, y en el Sur de Europa, en particular, las fiestas populares se han convertido en un fenómeno cultural de gran envergadura, especialmente asociado a la afirmación de las identidades autonómicas y locales, así como un movimiento de revitalización de las tradiciones, que es también común al ámbito europeo (Boissevan, 1992). Ello se explica, en parte, porque la fiestas modernas se constituyen como celebraciones reflexivas de la identidad, como una puerta de acceso a la trascendencia de la propia cotidianidad y como la emergencia de un tiempo especial para la recuperación del sentido en un contexto social secularizador y destradicionalizador (Ariño Villarroya y Gómez, 2012). Así, la fiesta, como manifestación que es de la llamada cultura popular, aglutina las más diversas formas de creatividad, constituyendo de este modo un producto social complejo, paradójico y dialéctico, dentro del cual es posible descubrir, no solo los mecanismos que impulsan hacia lo común, lo solidario, lo sociable y lo altruista (Antebi y Pujol, 2008), sino también, una "cara más oscura" que nos revela las tensiones y correlaciones entre fuerzas sociales; entre valores dominantes e impugnaciones colectivas; entre la estructura y la comunidad donde el poder intenta legitimarse para preservar el orden existente (Delgado, 2003). Por todo ello se puede hablar de cultura festiva, en la medida que constituye un producto específico de la cultura popular en términos amplios, es decir,

la política cultural y su relación en las pugnas por la hegemonía, la inclusividad social y la igualdad de género. En los tres casos se ha llevado a cabo una investigación cualitativa a partir de 9 entrevistas semi-dirigidas, a agentes festivos claves en la organización y desarrollo de las diferentes festividades, es decir, concejales de los consistorios, responsables y participantes de entidades festivas, así como, expertos en la cultura festiva local. Así pues, se ha desarrollado un análisis de contenido de los informes y memorias sobre la cultura festiva local de los gobiernos locales y de los organismos organizadores de las fiestas, además de un seguimiento de la prensa local en los tres casos estudiados. 
no como un objeto cultural fijado y legitimado sino como una expresión dinámica donde se advierten encrucijadas y préstamos con la cultura de masas 0 con la cultura de élite (García Canclini, 2002). Asimismo, podemos considerarla una manifestación de cultura "popular" en la que participan las clases subalternas y se expresan culturalmente en su relación con las clases dominantes, ya sea en términos de resistencia 0 consenso (Grignon y Passeron, 1989; Hernández i Martí, 2000).

Al mismo tiempo, la relación entre fiesta y patrimonio ejemplifica el creciente fenómeno de la patrimonialización de la cultura, al tiempo que ilustra la propia modernización de la fiesta (Hernàndez i Martí et al., 2005).

Las sociedades modernas y las fiestas tradicionales han tenido hasta entonces una relación que atraviesa diversas etapas. Anteriormente, en las sociedades feudales occidentales, impregnadas por el relato religioso cristiano, las fiestas eran ya eventos transcendentes que legitimaban los grandes poderes que las organizaban, como la monarquía, la iglesia 0 los consejos municipales, si bien en la fiesta tradicional siempre existieron actos, expresiones o ritos de contestación al poder, proliferando festejos de inversión del orden existente (el ciclo del carnaval). No obstante, las fiestas, como procesos rituales, están en constante transformación, convirtiéndose en espacios de cambio de los poderes establecidos. Siguiendo a V. Turner (1988) podemos afirmar que son "reinos de la posibilidad pura" donde se asoman modelos alternativos de sociedad, aun cuando las acciones rituales estén tuteladas según reglas firmemente establecidas por la tradición y la costumbre. Así pues, con la llegada de la burguesía al poder político, especialmente en la segunda mitad del siglo xIx, se llevó a cabo una reorganización del calendario festivo de acuerdo tanto con los nuevos valores capitalistas dominantes (Ariño, 1993). Como consecuencia de ello, se suprimieron numerosas fiestas tradicionales y se instituyeron nuevas celebraciones modernas. El avance del proceso de secularización, sobre todo a partir de los años sesenta del siglo xx, agudizó la destradicionalización festiva, al tiempo que reforzó las grandes fiestas urbanas, que el franquismo intentó instrumentalizar para legitimar el régimen dictatorial, generando una hegemoniza- ción conservadora y tradicionalista de muchas fiestas que llega, en parte, a nuestros días (Hernàndez i Martí, 1996).

Con la llegada de la democracia y el estado autonómico, en los años ochenta se produjeron tres fenómenos: se comenzó a experimentar un gran resurgir de las fiestas (Hernàndez i Martí et al., 2008), se evidenció un progresivo proceso de retradicionalización 0 revitalización turístico-patrimonial de fiestas decadentes y emergieron nuevos rituales festivos asociados a la celebración reflexiva de las identidades colectivas reconstituidas (García Pilan, 2010). Paralelamente, surge una auténtica política cultural festiva, diferente a las tradicionales mayordomías, mayoralías o comisiones municipales 0 eclesiásticas encargadas de organizar las viejas fiestas, pues se traducía en la constitución y consolidación de nuevos aparatos institucionales especializados en fiestas y en cultura popular, en concejalías, delegaciones, organismos autónomos y federaciones, incluso en empresas orientadas a la organización de eventos festivos. De este modo, los ayuntamientos se esforzaron en dinamizar la producción de fiestas, en aumentar los recursos a ellas destinados, en amplificar la trama institucional de las festividades, en diversificar los programas de actividades, en convertir las fiestas "de todos" en fiestas "para todos" (Velasco et al., 1996). A ello debe añadirse su preocupación por generar discursos que enfatizarán el carácter "participativo" e "integrador" de las fiestas, minimizando sus aspectos conflictivos o subversivos, e implementando mecanismos de control y ordenamiento del espacio público festivo (Antebi y Pujol, 2008; Delgado, 2003). Por otra parte, la aprobación por la UNESCO, en 1989, de la Recomendación para la Salvaguarda de la Cultura Tradicional y Popular, la celebración de la Convención de la UNESCO para la Salvaguarda del Patrimonio Inmaterial (2003) y la puesta en marcha de una lista oficial unificada de bienes inmateriales como Patrimonio de la Humanidad (2008) han contribuido a ratificar, institucionalizar y dinamizar todo aquello relacionado con el patrimonio festivo, que estaría plenamente inserto dentro del patrimonio inmaterial.

En todo caso debe subrayarse la centralidad de las federaciones 0 juntas coordinadoras de fiestas, 
Cultura festiva, política local y hegemonía social: Comparativa de los casos de los Moros i Cristians (Alcoi)...

que suelen mantener estrechos contactos con las instituciones públicas (a veces estas conceden subvenciones a las asociaciones festivas, generándose relaciones clientelares), con la iglesia, 0 con sectores comerciales, debido a la necesidad de financiación de las asociaciones. Sin embargo, a pesar de su relevancia socio-política y de la gestión de abundantes recursos públicos, estos organismos no han desarrollado hasta hace una década mecanismos de rendición de cuentas 0 de participación ciudadana directa como en otras áreas de la gestión cultural (Rius-Ulldemolins y Rubio, 2013). En este sentido, como mostraremos a continuación, este intento de desarrollo de una gobernanza local y del desarrollo de nuevas formas de participación más democráticas e inclusivas con todos los segmentos sociales, incluyendo a las personas inmigradas y la consecución de la igualdad de género festiva ha topado con notables límites generando, en ocasiones, conflictos sociales. Así, la fiesta se ha convertido en un "campo de batalla" entre los sectores que propugnan una modernización inclusiva y los sectores que la utilizan como una herramienta para conservar una hegemonía social y un orden social que reproduce la desigualdad en el acceso social a la fiesta y la discriminación de género (Bullen y Diez Mintegui, 2002), como veremos en los análisis de los casos de Moros i Cristians (Alcoi), de las Falles (València) y de la Patum (Berga) estudiadas a continuación.

\section{CULTURA FESTIVA, IDENTIDAD COLECTIVA Y GOBERNANZA LOCAL EN EL PAÍS VALENCIANO Y CATALUNYA: ANÁLISIS DE LOS CASOS DE LOS MOROS I CRISTIANS (ALCOI), LAS FALLES (VALÈNCIA) Y LA PATUM (BERGA)}

\section{Moros i Cristians (Alcoi): Inicio de cambio en una cultura festiva hegemonizada por una concepción tradicional del género}

Emplazada entre los parques naturales de La Mariola y La Font Roja se encuentra a Alcoi, en las comarcas centrales del País Valencià. Ciudad industrial, Alcoi fue puntera en el desarrollo de la industria textil, papelera y metalúrgica del estado español. Así, la entrada en la modernidad se caracterizó por grandes transformaciones sociales, económicas y políticas. Los nuevos valores sociales, junto a los sistemas de género nacientes, se proyectaron en las festividades alcoyanas donde los hombres burgueses, reunidos en asociaciones festivas, filaes, organizaron y crearon los códigos que rigen el ritual alcoyano, incluso hoy en día, y así, incrementando su valor, los envolvieron de prestigio y poder, convirtiéndolos en escaparates del poderío social de la masculinidad hegemónica (Gisbert Gracia, 2011).

La Fiesta de Moros y Cristianos de Alcoi, tal y como la conocemos a día de hoy, es una tradición creada en un contexto de modernidad, y como tal es una invención (Hobsbawn, 1998). Como tradición, es el resultado de una superposición de invenciones producidas a lo largo del tiempo. No se tratan, por supuesto, de creaciones arbitrarias, sino de manifestaciones concebidas por personas que actúan en función de intereses y pensamientos marcados por las circunstancias históricas propias del momento que viven, por su género, por su posición en la escala social y por su contexto cultural. Asimismo, según Antonio Ariño, el modelo alcoyano es "la matriz de la cual han brotado las diversas formas de dramatización cristiano-mora [en el sur del País Valencià]" (Ariño, 1988: 34). Sus rasgos característicos están marcados por ser fiestas patronales en honor a Sant Jordi con una estructura temporal de tres días y su estructura organizativa, como se describió anteriormente, está formada por filaes que, aunque disponen de autonomía festera, administrativa y rectora, no tienen personalidad jurídica, consecuentemente, son núcleos filiales de la Asociación de San Jorge (ASJ), una corporación de utilidad pública, sobre la cual el Ayuntamiento de Alcoi delega la organización festiva. En la Asamblea General de la ASJ, formada exclusivamente por hombres, "reside la suprema potestad de decisión e interpretación"”, es decir, desde ésta se crean to-

4 "En la asamblea general reside la suprema potestad de decisión e interpretación, y es de competencia el resolver toda cuestión de que deba conocer con arreglo al presente estatuto o que ese someta a su examen y deliberación" Art. 100 (Estatuto de la Asociación de San Jorge, 2016). 
das las codificaciones (implícitas y explícitas) que rigen las festividades alcoyanas, desde la estructura organizativa hasta los papeles que cada persona debe representar. Pero no solo crean y deciden las codificaciones, sino que también las interpretan, otorgándose a sí mismos una herramienta de poder muy potente dentro de la sociedad alcoyana, además de creación ideológica, de vehiculización y transmisión de valores.

En algunas celebraciones de Moros i Cristians se ha abierto la fiesta a la incorporación de la mujer en las filaes (Heuzé, 2003). Sin embargo, este no es el caso de Alcoi donde se sigue reproduciendo desde su origen un marcado carácter sexista de la fiesta (Martínez Pozo, 2015). De este modo, el papel otorgado a las mujeres en las festividades alcoyanas, se construyó conforme estereotipos tradicionales de "feminidad" de cuidadora 0 de mujer-objeto ${ }^{5}$ (Bullen y Diez Mintegui, 2002). Sin olvidar que son unas tradiciones desiguales y discriminatorias que, a pesar de presentarse bajo un discurso igualitario e integrador que incluye a las mujeres para el desarrollo del ritual, esconde una concepción machista que relega a la mujer a posiciones subalternas y pasivas (Hernández i Martí, 1996).

No obstante, esta característica se reveló en toda su dimensión de control hegemónico y de reproducción social a partir de la lucha de algunas mujeres para lograr una posición de igualdad real en la fiesta. En 1998, Nuria Martínez saltó a la luz pública, por convertirse en la primera mujer festera de las festividades de Moros y Cristianos de Alcoi. Esta iniciativa quebrantó la frontera simbólica que posicionaba a cada género en el lado que se les había asignado, tanto en el orden social como en el simbólico (Bourdieu, 2000). La ruptura del margen

5 Este segundo modelo de feminidad se introdujo en las festividades alcoyanas a finales de los años ochenta, con dos fines concretos, el primero como estrategia masculina de desviar el interés que algunas alcoyanas empezaban a mostrar por participar activamente en las festividades y el segundo, para añadir espectacularidad y fastuosidad al desfile de los ejércitos. Este tipo de feminidad hace hincapié en el aspecto físico y alienta la imagen estereotipada de la feminidad oriental pensada por hombres occidentales como misteriosa, exótica y erotizada (Edward Said, 2004). simbólico trajo consigo un conflicto socio-festivo que, a día de hoy 20 años después, todavía divide a la población alcoyana en dos posiciones: la primera, defiende la tradición, entendida como inmanente e inmutable y está abanderada por el entramado organizativo de la fiesta, con la ASJ a la cabeza. Desde la segunda posición, se aboga por un cambio en las estructuras de los rituales para conseguir la participación de las mujeres con plena igualdad. Esta segunda posición está representada por una asociación de mujeres y hombres creada ad hoc en el año 2003, la Associació Fonèvol.

Las estrategias de acción para el cambio que ha llevado a cabo la Associació Fonèvol en los últimos 20 años se han ubicado principalmente en el ámbito público y civil de la sociedad alcoyana, con campañas de concienciación y visibilización de la situación real de las mujeres en las rituales, mediante la organización de jornadas y campañas coeducativas en los centros educativos de la ciudad y a través de la publicación de artículos de opinión en la prensa local. Pero, además, conscientes del papel central que jugaban las instituciones públicas y políticas de la ciudad en la mediación y la resolución del conflicto, las personas integrantes de Fonèvol se han entrevistado, en no pocas ocasiones, con el gobierno municipal y con otros partidos políticos del consistorio, además de presentar mociones en los plenarios y de solicitar el amparo del gobierno local en diferentes ocasiones.

Las respuestas obtenidas durante los primeros años del conflicto en los que el ayuntamiento estaba liderado por el Partido Popular, defensor férreo del posicionamiento tradicionalista, fueron negativas, llegando incluso a ser criticada esta actitud por el Síndic de Greuges (Defensor del Pueblo) por hacer caso omiso a sus recomendaciones para facilitar la integración de las mujeres en el ritual alcoyano. № obstante, la situación ha evolucionado favorablemente desde el cambio de gobierno local que tuvo lugar en el año 2011, donde la ciudad pasó a ser gobernada por el partido socialista (PSOE). A lo largo de estos siete años se ha construido una relación de colaboración entre el gobierno local y la Associació Fonèvol. Esta cooperación se ha visto plasmada en el último Plan de Igualdad aprobado por unanimidad por el consistorio alcoyano, en el que uno de los 
Cultura festiva, política local y hegemonía social: Comparativa de los casos de los Moros i Cristians (Alcoi)...

objetivos previstos es conseguir la integración real y efectiva de las mujeres en las festividades locales. Pero a día de hoy esta igualdad real y efectiva todavía no se ha materializado en el ritual.

No obstante, es cierto que el año 2015 fue un punto de inflexión del proceso que acabamos de describir. Durante el mes de abril la Associació Fonèvol pudo participar públicamente en la fiesta por primera vez, organizando la primera entraeta (entrada a la fiesta) mixta de la historia alcoyana. En esta ocasión cerca de un centenar de personas, la mayoría mujeres, desfiló por las calles del centro de la ciudad, recibiendo los aplausos de apoyo del público. También el 22 de abril la primera esquadra compuesta por festeras de pleno derecho de la Filà Marrakesch participó en un acto oficial del ritual. Sin lugar a dudas, estos dos actos representan una grieta en el control hegemónico de la fiesta y el inicio prometedor de una dinámica más inclusiva y menos discriminatoria.

\section{Falles (València): Una neo-tradición con dificultades para evolucionar}

Actualmente, les Falles constituyen una de las fiestas más importantes del mundo, con una masiva afluencia de visitantes y una red asociativa fallera de más de 100000 personas, en la ciudad de València, tercera ciudad del Estado español con más de 790 mil habitantes en 2016. Ello es debido a que las fiestas de las Fallas tienen una larga trayectoria, con importantes mutaciones a lo largo de los años, que comienza con los populares catafalcos satíricos levantados hacia mediados del siglo xVIII en las plazas y calles de los barrios históricos de la ciudad. Entre esta época y 1936 las Fallas se convirtieron en la primera fiesta de la ciudad, desplazando al Corpus Christi, y en una de las más importantes y carismáticas del País Valenciano. Ya antes de la Guerra Civil las Fallas representaban una auténtica liturgia civil del valencianismo temperamental que expresaba emocionalmente la identidad valenciana (Ariño Villarroya, 1992). De este modo, y tras ser inicialmente perseguidas por las autoridades burguesas, cuando éstas descubrieron que las podían instrumentalizar a su favor, las Fallas se convirtieron en la máxima expresión sentimental de Valencia, a modo de nueva religión civil local (Ariño Villarroya, 1992).

Este hecho explica que, con la victoria franquista, el nuevo régimen procedió a intensificar el control de la fiesta fallera mediante una red de disposiciones, reglamentaciones e instituciones específicas, las cuales acabaron con el anterior pluralismo político e ideológico de la fiesta y la convirtieron en una expresión propagandística de las directrices del nuevo régimen (Hernàndez $\mathrm{i}$ Martí, 1996). Con ello se inauguró un largo periodo en el que la fiesta se convirtió en una herramienta de la hegemonía en la vida política y social de la ciudad. Así, bajo el franquismo, las Fallas fueron reinventadas en profundidad, pese a las resistencias desde el mundo fallero (Hernàndez i Martí, 1998). Por ello, a la hora de producirse la transición democrática, las Fallas ya constituían un producto cultural con unas enormes potencialidades de uso político, como efectivamente sucedió. Así, y ante la perplejidad y pasividad de las fuerzas de izquierda, las Fallas fueron reinstrumentalizadas por la derecha postfranquista local, reconstituida en el Ilamado movimiento del blaverismo — secesionismo cultural y lingüístico de carácter anti catalanista y españolista- (Flor, 2011), vertebrando a través del universo fallero sus mensajes antiprogresistas, que recibieron así un extenso apoyo popular en el marco de la nueva democracia constitucional (Peris Llorca, 2014).

Durante los últimos treinta años, las Fallas han seguido su expansión imparable por la ciudad de Valencia y por multitud de poblaciones del País Valenciano, aumentándose el número de fallas en más de 400 comisiones y generando un significativo impacto económico valorado en 612 millones de euros anuales, el 82,7\% en el sector hostelero y turístico, calculándose un millón de visitantes durante la fiesta (Interagrupación de Fallas, 2007). También es cierto que las fiestas recibieron la importante cantidad de 4,7 millones de euros por parte del Ayuntamiento en 2016 (Ajuntament de València, 2016). Desde sus inicios, las Fallas han experimentado grandes metamorfosis, siendo la última un acusado proceso de patrimonialización institucional y civil (Hernàndez, Santamarina, Moncusí 
y Albert, 2005), transformándose la celebración ritual y la vivencia social de la fiesta, aspecto que le confiere una potencialidad y un atractivo como posible recurso turístico.

A pesar de ello, este proceso de institucionalización y masificación no ha ido acompañado de un completo proceso de democratización de la fiesta. Así, la ciudadanía de Valencia está conformada por un sector fallero muy organizado (el $6,5 \%$ de la población adulta), aunque entre los no falleros también existe un sector anti-fallero usualmente ignorado. Esta división se explica en gran medida por la instrumentalización de la fiesta por parte de los sectores tradicionalistas y conservadores. Una utilización política que ya fue iniciada por el franquismo y canalizada por las fuerzas más reactivas a la democracia durante la transición, especialmente por los movimientos anticatalanistas (Hernàndez i Martí, 1996). Aunque no se puede negar la existencia de una diversidad interna en el mundo de las fallas, las comisiones falleras organizadas al modo convencional hegemonizan y co-organizan una parte de la actividad festiva de la ciudad, produciéndose el fenómeno de la fallerización del calendario festivo e indirectamente de la vida social de la ciudad.

Las Fallas conforman un macro evento organizado. Las comisiones falleras se constituyen en agrupaciones y federaciones, gobernadas por la Junta Central Fallera (JCF), creada en 1939 por el régimen franquista para controlar la fiesta de base. La JCF es un organismo autónomo municipal, presidido por el concejal de Fiestas y el alcalde/sa, que ha seguido instrumentalizando la fiesta bajo la influencia de los sectores políticos más conservadores de la ciudad (Hernàndez, 2011). Con todo, la sociabilidad fallera presenta unas características de gran antigüedad, uniformidad, persistencia, densidad, estabilidad y multiactividad (Hernàndez, 2014) que cohesiona ciertos sectores de la ciudad (X. Costa, 2002). Sin embargo, durante el franquismo se realiza una lectura tradicionalista y religiosa de la fiesta en la que se privilegia como acto central la gigantesca Ofrenda de Flores a la Virgen de los Desamparados, creada por el régimen en 1945, si bien posteriormente evolucionó hasta convertirse en un gigantesco y secularizado desfile de 110000 personas durante dos jornadas, en el que el colecti- vo fallero muestra su fortaleza y vitalidad (Hernàndez i Martí, 1996).

Desde la recuperación de la democracia hasta 2015 las Fallas han contado con un férreo control municipal con un planteamiento vertical e intervencionista en el cual la concejalía de Fiestas y de Cultura Popular ha contado con un gran peso simbólico y político en el gobierno municipal. Este peso se explica por su posición clientelar y paternalista hacia el colectivo fallero que, a cambio de una financiación generosa y un escaso control en su ocupación del espacio público, constituye una apetitosa bolsa de votos y una importante frente de legitimación simbólica del poder municipal, que explican, en parte, un periodo ininterrumpido de gobiernos conservadores y de una sola alcaldesa, Rita Barberá, durante veinticinco años. Asimismo, se constata la reproducción de un discurso oficial inmovilista durante estos años que insistía en que, las Fallas son "la mejor fiesta del mundo", promocionándose prácticamente por sí solas y no necesitaban cambios para integrar a las nuevas personas inmigrantes 0 dar un rol más inclusivo y abierto socialmente a la fiesta.

A diferencia de los cambios sufridos por las Fallas desde sus inicios, el rol asignado a las mujeres continúa todavía influido por la visión tradicionalista hegemónica de la fiesta formulada en pleno franquismo. Entre los papeles de las mujeres, destaca la pasividad ritual de la Fallera Mayor, la cual está limitada a expresar en público sus opiniones a ciertos actos festivos y debe vestir y comportarse según un rígido canon de "buenas prácticas". Aunque con dificultades se han producido algunos avances en la participación de las mujeres en las tareas ejecutivas de las comisiones, y la candidatura de la UNESCO ha introducido un cierto discurso modernizador en relación a la multiculturalidad y el papel de las mujeres en las festividades ${ }^{6}$ no se ha

6 En el informe de la concesión del comité intergubernamental de la UNESCO (11.COM 10.B.30) se afirmaba que: "Participation and belonging to a Fallas Commission is open to any social group, including men and women of all ages, professions, social class or geographical or cultural background. The festivity is said to have evolved to allow for a growing representation and participation of women in its planning and conduct" (UNESCO, 2016). 
Cultura festiva, política local y hegemonía social: Comparativa de los casos de los Moros i Cristians (Alcoi)...

transformado en lo esencial, es decir, la dinámica de organización y desarrollo de la fiesta. Como lo demuestra el hecho de que, del total de 22 presidentes/as de agrupaciones falleras, solamente dos son mujeres (Junta Central Fallera, 2017), o que en 2017 solo el 11,2 \% de las comisiones están presididas por mujeres, lo que evidencia la fuerte masculinización de las estructuras de poder falleras. Además, en el Reglamento Fallero todavía vigente (es de 2002), se especifica el vestido de las falleras y la prohibición de uso de "piezas masculinas"7.

Ciertamente, el nuevo gobierno local de izquierdas, elegido en el 2015, ha intentado modificar las estructuras, los reglamentos y las dinámicas organizativas. En su programa electoral, la Coalició Compromís, izquierda valencianista, apostaba por una gestión rigurosa, con participación directa del mundo fallero (rompiendo el esquema representativo y clientelista), sostenible y con una voluntad de transformación progresista de la fiesta, ya que la juzgaba demasiado anquilosada en ciertas inercias y en parámetros conservadores (Coalició Compromís, 2015). Dos años después, su acción de gobierno en la nueva concejalía de Cultura Festiva ha alcanzado significativos éxitos en la modernización de la marca y de la gestión de la fiesta. Sin embargo, el avance hacia la democratización y la transparencia en la gestión, así como en la inclu-

7 Según el capítulo II del reglamento fallero sobre la indumentaria, el artículo 64 específica que "Con el fin de dar el mayor realce y esplendor a los actos y desfiles falleros, las comisiones velarán para que en la fiesta de las Fallas se utilice la indumentaria fallera y valenciana tradicional, diferenciando la utilizada por el hombre de la usada por la mujer valenciana y fallera: a) el fallero utilizará el traje instituido en el IV Congreso General Fallero 0 cualquier traje tradicional valenciano. b) La fallera deberá lucir el tradicional traje de Valenciana 2. Queda terminantemente prohibida la utilización de piezas que no sean acordes a la indumentaria tradicional fallera expresada anteriormente, asimismo no se permitirá la utilización de piezas masculinas por falleras, en actos oficiales, con independencia del cargo que ocupan. A estos efectos, se considera el blusón 0 blusa prenda masculina" (Junta Central Fallera, 2017). Como podemos ver la indumentaria sólo se refiere a las mujeres y, específicamente, para prohibir y, por lo tanto, limitar su libertad de actuación según unos cánones claramente sexistas. sividad social y la ruptura de la desigualdad de género, está siendo una tarea costosa. Iniciativas como la de un congreso fallero 0 han encontrado una persistente resistencia cuando no una abierta hostilidad por parte de los sectores más conservadores (Garsan, 2017). Estos sectores, liderados por la Interagrupación de Fallas, se han convertido en un factor de desgaste del gobierno local actuando como extensiones la derecha españolista que concibe el sector fallero como un puntal de su estrategia de hegemonía política y social, amenazada por la descomposición originada por los numerosos casos de corrupción (Rius-Ulldemolins et al., 2017), pero que ambiciona la recuperación del poder en las próximas elecciones locales con un programa de mantenimiento del orden tradicional en la fiesta.

\section{La Patum de Berga: intentos de modernización e inclusividad de la fiesta desde el gobierno local}

La Patum se desarrolla en Berga, ciudad de 16 mil habitantes y capital de la comarca del Berguedà (Catalunya). Enclavada en el Pre-Pirineo se encuentra cerca del curso del río Llobregat que comunica con la Catalunya central y con Barcelona $y$, por ello, ha tenido una significativa componente industrial en su economía y un flujo de población inmigrante durante el siglo xx. Plaza fuerte de los carlistas durante la Primera y la Tercera Guerra Carlista, así como del movimiento obrero anarquista, la ciudad se ha caracterizado por un fuerte sentido de identidad local y un potente dominio del catalanismo en el siglo $\mathrm{xx}$, y del independentismo en el siglo xxı que, en 2015, superó el $80 \%$ de los votos (Generalitat de Catalunya, 2015). Por ello, la ciudad ha sido gobernada desde la recuperación de la democracia por la derecha del Partit Demòcrata Europeu Català (antes Convergència i Unió) con la sola excepción de un gobierno socialista entre 2003 y 2007. Sin embargo, en las elecciones de 2015 ganó la candidatura de Unidad Popular (CUP), independentistas de izquierda, que ostenta desde entonces la alcaldía. En este contexto, la fiesta se ha convertido en un ámbito de disputa por la hegemonía entre los nuevos gobernantes y la oposición, 
que han defendido concepciones de la fiesta significativamente contrastadas.

La fiesta de La Patum es una celebración derivada de las procesiones medievales del Corpus y se estima que se celebra desde el siglo xv. Se celebra durante cinco días de la semana de Corpus en el que los Plens, con la combinación de bailes tradicionales y pirotecnia en la plaza de Sant Pere, constituyen los momentos centrales. En su codificación y reinvención como tradición durante el siglo xIx, como indica su cambio de nombre de Bulla por Patum, la fiesta incorporó elementos precristianos de la regeneración de la naturaleza y el ciclo de la primavera, disponiendo escenificaciones de la lucha entre moros y cristianos y la liberación del dominio feudal simbolizada por el águila real. Una transformación de la fiesta que se produce a lo largo del siglo xIx con la incorporación escalonada de las comparsas. A través de este proceso se puede observar una enriquecedora mutación festiva mediante la introducción de gigantes y enanos fabricados como representaciones simbólicas y con la composición de las músicas típicas de la fiesta (Felipó, 2005). La importancia de la festividad analizada, viene siendo reconocida desde los años ochenta a nivel autonómico (fue declarada el 1983 como Fiesta patrimonial de interés nacional en Catalunya) e internacional, obteniendo en 2005 el reconocimiento ya mencionado de la UNESCO, potenciando aún más su dimensión central a nivel local y como atracción turística.

Dado esta creciente importancia, desde 2001 la fiesta está gestionada por el Patronat Municipal de La Patum, que se constituye en órgano de gobernanza y que tiene por objeto como afirma su primer artículo: "administrar, conservar y coordinar el desarrollo de la fiesta de La Patum, declarada Obra Maestra del Patrimonio Oral e Inmaterial por la UNESCO; todas las actividades derivadas y relacionadas estrictamente con las comparsas de la fiesta, sus actuaciones y sus integrantes; velar por la buena utilización del nombre y la imagen de la celebración; así como todo lo relacionado con su imagen corporativa, publicitaria y de difusión y todo aquello que, de forma general 0 específica, incluya el nombre Patum 0 haga referencia" (Ajuntament de Berga, 2016). El patronato estructura la participación en la fiesta de ocho colectivos o comparsas: Tabal, Plens, Nans Vells, Nans Nous, Àngels, Àliga, Guita Xica y Guita Grossa. Estas a su vez están compuestas por una junta rectora y su composición muchas veces estaba conformada por liderazgos largos y fuertes y ligada a varias familias de la ciudad que "heredan" informalmente el cargo. Por otra parte, su composición ha sido objeto de críticas por su carácter cerrado y por el predominio de hombres y la marginación de las mujeres en los puestos de dirección de la fiesta: por ejemplo, de 2007 a 2011, del total de 19 personas que conformaban el patronato, 16 eran hombres (Patronat Municipal de La Patum, 2017). De hecho, según la regidora de fiestas, Mònica Garcia, "La Patum es un ámbito dominado por los hombres. Y estaban acostumbrados a la interlocución con hombres. Nunca había habido una alcaldesa 0 una concejala de Patum" (Regió7, 2017).

Uno de los puntos centrales de la fiesta es la participación en los Plens, los llamados salts (en castellano, saltos), en los cuales participan los miembros de las comparsas, unas 280 personas en cada uno y unas decenas de personas que eran escogidas por las mismas comparsas y por el ayuntamiento de una forma clientelar y opaca. Esta gestión oligárquica de un bien escaso, considerado como uno de los mayores honores para los berguedans y berguedanes, ha sido objeto de una intensa polémica política y es uno de los temas que más debate genera en los plenos de la corporación local (Morales, 2017b). En relación a la cuestión del género y a la promoción de la participación de la mujer el 2015, el ayuntamiento avanzó en la igualdad al nombrar en el Patronat a seis mujeres de los ocho que el consistorio tiene como representantes en el patronato de la fiesta (Ajuntament de Berga, 2015). Esta decisión, unida a la de ampliación del patronato que se renueva cada año ha facilitado que se ampliase el número de mujeres a 10 de un total de 31 , con lo que, aunque siguen siendo minoría, el porcentaje ha aumentado del $15 \%$ de 2007 2011 a un $32 \%$ entre 2015-2017.

Este creciente intervencionismo del ayuntamiento se ha reflejado, además, en el conflicto que se generó por la expulsión de tres miembros 
Cultura festiva, política local y hegemonía social: Comparativa de los casos de los Moros i Cristians (Alcoi)...

de la comparsa de Plens y de Maces, que criticaron abiertamente a la dirección de las comparsas por clientelismo y marginación de las mujeres. En este sentido, el ayuntamiento medió para que se readmitiesen a los miembros expulsados acusados de "sedición" y recibieron por parte del sector conservador de la fiesta -y próximo a la derecha local— acusaciones de traicionar la fiesta (Regió7, 2017). Esta polémica saltó además a la arena pública local, al presentarse al concurso de los carteles una propuesta crítica acerca de la gestión tradicional de la fiesta. Con el título Diàleg (diálogo) el artista quería denunciar lo contrario, es decir, la ausencia de transparencia y las barreras a la participación, especialmente para las mujeres en la fiesta, mostrando una mujer delante de una pared (Morales, 2017a). En pleno conflicto, el ayuntamiento tomó el control de las dos comparsas y, además, destituyó a sus jefes por no haber redactado los estatutos, como sí hicieron las otras comparsas, para así formalizar las reglas de participación y elección de los jefes. Una redacción de los estatutos que ha puesto la legitimidad tradicional en duda y que ha generado un proceso participativo y de cambio dentro de la fiesta. En este sentido la racionalización y la democratización de la tradición han ido de la mano.

Incidiendo en esta dinámica democratizadora e igualitarista, el nuevo equipo de gobierno decidió en el 2016 reformar el sistema de distribución en los Plens mediante un sistema mixto en el que se otorga una gestión de la participación a las comparsas y un sistema abierto gestionado mediante un sorteo público y transparente, previa inscripción en el ayuntamiento o telemáticamente. Asimismo, se pedía como condición únicamente mostrar el documento nacional de identidad 0 bien la inscripción en el padrón municipal, con lo que se abría la participación explícitamente a inmigrantes no regularizados. Esta decisión respondía a la intención de combinar el reconocimiento de la participación de las comparsas, que podían distribuir 10 salts cada una, junto con la promesa electoral que responde a la reivindicación de sectores de la fiesta que apostaban por una mayor accesibilidad y por una distribución más equitativa. Por su parte, los concejales del gobierno de la CUP renunciaban a sus "privilegios" en los salts, pero conservaban este privilegio para los regidores de la oposición y para personal del consistorio.

Este cambio supone una transformación radical de la distribución tradicional y fue criticada por parte de los sectores conservadores como un ataque a la tradición, como una invasión de "forasteros" (al poder optar a los salts residentes de fuera de la ciudad) y como un riesgo para la fiesta, al permitir entrar a personas sin un conocimiento previo. Sin embargo, desde el ayuntamiento se ha insistido en el carácter inclusivo y "justo" — permitiendo un acceso individual y anónimo - de este método que supera la opacidad y clientelismo con el que se realizaba el acceso a los salts, que dependían de la voluntad del jefe de comparsa (Regió7, 2017). Como vemos, se han introducido elementos modernizadores y democratizadores en la fiesta de La Patum, pero siguen existiendo numerosas resistencias e inercias en la distribución, que sigue en parte siendo oligárquica. En este juego, la derecha tradicionalista del PDECAT está utilizando este conflicto para generar un debate permanente en las reuniones del ayuntamiento, para erosionar el gobierno de la CUP en los medios de comunicación y para retomar la alcaldía en las próximas elecciones (Morales, 2017b).

\section{CONCLUSIONES}

La importancia de las tradiciones en las sociedades avanzadas ha sido subestimada 0 bien se le ha atribuido un carácter residual. Frente a estas posiciones algunos autores han destacado su rol cohesionador y vertebrador de las tradiciones en el mundo social moderno (Shils, 2006). En este contexto, la cultura festiva de raíz tradicional, en proceso de patrimonialización y como elemento de festivalización de la cultura y de branding territorial, se ha revelado como un elemento hasta cierto punto dinamizador e innovador a nivel económico, turístico y de la sociabilidad en contextos urbanos (Ariño Villarroya, 1992; X. Costa, 2002; X. Costa, 2001). Sin embarg0, siendo ciertos estos aspectos la dimensión política, su rol en la dominación y en la reproducción de una sociedad desigual ha sido poco estudiada. Por ello, 
en este artículo hemos analizado tres casos festivos muy relevantes a nivel local e internacional cómo la cultura festiva ha sido instrumentalizada por parte de los sectores dominantes para conservar la hegemonía y para reproducir los patrones de sociabilidad tradicionales como la discriminación de género o la baja inclusividad social.

Estos tres casos nos muestran cómo en las sociedades modernas que han heredado, conservado, transformado y promovido una cultura festiva, ésta se convierte en un foco central -y no secundario, como se podría valorar desde una perspectiva de un materialismo determinista - de la política y la lucha por la hegemonía local. Ello lo convierte en arena de conflicto y en un factor independiente en la explicación de la reproducción social local. Y explica también la creciente consciencia de los sectores políticos locales orientados, por una parte, a competir por la hegemonía en la fiesta para mantener el control por parte de los sectores festivos tradicionalistas y, por otra parte, el reto de los gobiernos de izquierda para disputar los organismos de gobernanza de la fiesta a fin de promover un cambio inclusivo e igualitario. Esta disputa, en cada contexto local se expresa de forma distinta como puede observarse en los Moros i Cristians de Alcoi, principalmente en la reivindicación por la inclusión de las mujeres en la fiesta; en las Falles de València, en la tensión entre el nuevo gobierno y un sector festivo inmovilista y ultraconservador que bloquea en cierta medida el cambio; y en La Patum de Berga, en la que una decidida actuación del nuevo gobierno de izquierda ha conseguido una mayor inclusividad en la fiesta. Por finalizar, remarcar que los tres casos nos muestran un proceso vivo y en evolución que abre una vía de investigación y reflexión sobre el "lado oscuro" de la cultura festiva, en el sentido de pugna por la dominación y la hegemonía. El escenario contemplado en estos casos elegidos deberían ser estudiados en todas las fiestas, ferias, festivales 0 carnavales en las que se pueden estar desarrollando estos procesos de pugna entre la reproducción de las tradiciones y sus supuestas esencias y el fomento de cambios inclusivos e igualitarios en la cultura festiva y, por extensión, de la comunidad local.

\section{REFERENCIAS BIBLIOGRÁFICAS}

Ajuntament de Berga. (2015). Proposta de resolució. nomenament de representants de la corporació a órgans col.legiats que siguin competència del ple. Berga: Ajuntament de Berga.

Ajuntament de Berga. (2016). Estatuts del patronat municipal de la patum. Berga: Ajuntament de Berga.

Ajuntament de València. (2016). Pressupost municipal 2016. València: Ajuntament de València.

Antebi, A., y Pujol, A. (2008). Entre el poder y la máscara. una etnohistoria del carnaval de barcelona. Barcelona: Generalitat de Catalunya.

Ariño Villarroya, A. (1992). La ciudad ritual: La fiesta de las fallas. Barcelona: Anthropos.

Ariño Villarroya, A., y Gómez, S. (2012). La festa mare. les festes en una era postcristiana. Museu Valencià d'Etnologia-Diputació de València: València.

Ariño, A. (1988). Festes, rituals i creences. València: Edicions Alfons El Magnànim.

Arroyo Menéndez, M., Cabrera Sánchez, J. (2011). Dinámicas del cambio cultural en España: Explorando tendencias generacionales. Revista Española De Sociología, 15, 47-74.

Associació de Sant Jordi.Estatuto de la associación de San Jorge y ordenanza de la fiesta. Alcoi: Associació de Sant Jordi.

Ayuntamiento de Zaragoza. (2014). Las fiestas mayores en seis ciudades de España. origen, actividades y financiación. Zaragoza: Observatorio de Cultura. Ayuntamiento de Zaragoza.

Boissevan, J. (ed.). (1992). Revitalizing european rituals. Routledge: London.

Bourdieu, P. (1980). L'identité et la représentation. éléments pour une réflexion critique sur l'idée de région. Actes De La Recherche En Sciences Sociales, 35 (1), 63-72.

Bullen, M., y Diez Mintegui, C. (2002). Violencia y cambio de culturas androcéntricas. XI Congrés d'Antropologia FAAEE, (1), 20.

Burawoy, M. (2012). The roots of domination: Beyond bourdieu and gramsci. Sociology, 46 (2), 187-206. doi:10.1177/0038038511422725

Coalició Compromís. (2015). Programa de govern compromís 2015-2019. València: Coalició Compromís. 
Cultura festiva, política local y hegemonía social: Comparativa de los casos de los Moros i Cristians (Alcoi)...

Cohen, S. (2013). Musical memory, heritage and local identity: Remembering the popular music past in a european capital of culture. International Journal of Cultural Policy, 19 (5), 576-594. doi:10.1080/10286632.2012.676641

Connolly, M. G. (2011). The 'Liverpool model(s)': Cultural planning, liverpool and capital of culture 2008. International Journal of Cultural Policy, 1-20. doi:10.1080/10286632.2011.638982

Costa, X. (2002). Festive traditions in modernity: The public sphere of the festival of the'Fallas'in Valencia (Spain). 50 (4), 482-504.

Costa, X. (2001). Festivity: Traditional and modern forms of sociability. Social Compass, 48 (4), 541-548.

Delgado, M. (2003). Carrer, festa i revolta. Usos simbòlics de l'espai públic a Barcelona, 1951-2000. Barcelona: Generalitat de Catalunya.

Dowling, M. (2008). Fiddling for outcomes: Traditional music, social capital, and arts policy in northern ireland. International Journal of Cultural Policy, 14 (2), 179-194. doi:10.1080/10286630802106359

Ekinci, Y., y Hosany, S. (2006). Destination personality: An application of brand personality to tourism destinations. Journal of Travel Research, 45 (2), 127-139. doi:10.1177/0047287506291603

Felipó, R. (2005). La Patum, el Corpus Christi de Berga. Tarragona: Edicions El Mèdol.

Flor, V. (2011). Noves glòries a Espanya. anticatalanisme $i$ identitat valenciana. Catarroja-Barcelona: Afers.

García Canclini, N. (2002). Culturas populares en el capitalismo. México: Grijalbo.

García Pilan, P. (2010). Tradición en la modernidad avanzada: La semana santa marinera de Valencia,. Valencia: Museu Valencià d'Etnologia.

Garsan, C. (2017). Brexit fallero: Fuset se aleja de junta central mientras se hornea el congreso Carlos Garsán. Valencia Plaza, 27/06/2017

Generalitat de Catalunya. (2015). Resultats electorals eleccions municipals 20015. Berga. Barcelona: Departament de Governació, Administracions Públiques i Habitatge. Generalitat de Catalunya.

Giori, P. (2012). Hacer castells, construir nación. castells, modelo festivo y catalanismo. Trabajo final de máster en comunicació i estudis culturals. Girona: Universitat de Girona.

Gisbert Gracia, V. (2011). Feminidades y masculinidades en la fiesta de Moros y Cristianos de Alcoi. Prisma Social, 7, 92-119.

Gligorijevic, J. (2014). World music festivals and tourism: A case study of Serbia's guca trumpet festival. International Journal of Cultural Policy, 20 (2), 139-154. doi:10.1080/10286632.2012. 743531

Gramsci, A. (2011). ¿Qué es la cultura popular? València: Publicacions de la Universitat de València.

Gray, C. (2002). Local government and the arts. Local Government Studies, 28 (1), 77-90.

Gray, C. (2010). Analysing cultural policy: Incorrigibly plural or ontologically incompatible? International Journal of Cultural Policy, 16 (2), 215-230. doi:10.1080/10286630902935160

Grignon, C., y Passeron, J. (1989). Le savant et le populaire: Misérabilisme et populisme en sociologie et en littérature. Paris: Gallimard: Seuil.

Hernàndez i Martí, G. (1996). In Afers (ed.), Falles i franquisme a València. Catarroja-Barcelona.

Hernàndez i Martí, G. (2000). Les transformacions de la cultura popular. Afers, 37, 751-760.

Hernàndez i Martí, G. (1996). Falles i franquisme a València. Afers: Catarroja-Barcelona.

Hernàndez i Martí, G. (1998). Microidentidades colectivas: El caso de la fiesta en el país valenciano. VI Congreso Español de Sociología, A Coruña.

Hernàndez i Martí, G. (2016). Cultura festiva, identidad colectiva y política cultural. En J. Rius Ulldemolins, \& J. A. Rubio Arostegui (eds.), Treinta años de políticas culturales en España. participación cultural, gobernanza territorial e industrias culturales (pp. 231-248). València: Publicacions de la Universitat de València.

Hernàndez i Martí, G. Albert, M. Gómez Nicolau, E. et al. Requena, M. (2014). La cultura como trinchera. la política cultural en el país valenciano (1975-2013). València: Universitat de València.

Hernàndez i Martí, G. Moncusí, A. et al. Santamarina, B. (2008). Patrimonio etnológico e identidades en España. un estudio comparativo a través de la legislación. Revista Experimental de Antropología, 8 (207), 223. 
Hernàndez i Martí, G. Santamarina, B. Moncusí, A. et al. Albert, M. (2005). La memoria construida. patrimonio cultural y modernidad. Valencia: Tirant lo Blanch.

Hernàndez, G. (2011). Focs de falla. articles per al combat festiu. València: Obrapropia.

Heuzé, L. (2003). La incorporación de las mujeres en las fiestas: Las negras de Petrer (Alacant). En M. Albert-Llorca, \& J. González Alcantud (eds.), Moros y cristianos. representaciones del otro en las fiestas del Mediterráneo occidental (pp. 103-114). Granada: Diputación de Granada.

Hobsbawn, E. (1998). Introducció: L'invent de la tradició. En E. Hobsbawn, \& T. Ranger (eds.), L'invent de la tradició (pp. 5-27). Vic: Eumo.

Interagrupación de Fallas. (2007). Estudio del impacto económico de las fallas. València: Interagrupación de Fallas-Ajuntament de València.

Jeanpierre, L. (2011). Bourdieu ou gramsci? une fausse alternative pour les études culturelles. En P. Casanova (ed.), Des littératures combatives. L'internationale des nationalismes littéraires (pp. 73-95). Paris: Raisons d'agir.

JuntaCentralFallera. (2017). Presidents d’Agrupacións. Ilistat de presidents d'agrupacions de junta central fallera. València: Junta Central Fallera.

Leoussi, A. S. (2013). Max weber in the thought of edward shils (1910-1995) and ernest gellner (1925-1995): The paradox of two weberian approaches to the understanding of nations and nationalism? Ethnic and Racial Studies, 36 (12), 1957-1976. doi:10.1080/01419870.2 012.681674

Martínez Pozo, M. Á. (2015). La mujer en las fiestas de Moros y Cristianos. Revista de Antropología Experimental, 15, 79-87.

Merli, P. (2013). Creating the cultures of the future: Cultural strategy, policy and institutions in gramsci. International Journal of Cultural Policy, 19 (4), 399-420. doi:10.1080/10286632.20 11.643872

Morales, A. (2017a). Jordi plana: «Jo no vaig fer un cartell perquè anunciés la patum, sinó per denunciar un fet». Naciodigital, 10/06/2017.

Morales, A. (2017b). La patum escalfa l'ambient al ple de Berga, que s'omple de crítiques i retrets. Naciodigital, 02/06/2017.
Patronat Municipal de La Patum. (2017). Memòries Patronat municipal de La Patum, 2000-2016. Berga: Ajuntament de Berga.

Peris Llorca, J. (2014). Populismo y literatura popular. La función de las fallas de Valencia en la extensión del blaverismo. Pasajes: Revista de Pensamiento Contemporáneo, 46, 42-61.

Putnam, R. D. (ed.). (2002). El declive del capital social: Un estudio internacional sobre sociedades y el sentido comunitario. Barcelona: Galaxia Gutemberg.

Regió7. (2017). «Repartir els salts de plens de Patum a través d'un sorteig és una fórmula justa» Mònica Garcia, regidora de Patum, respon les preguntes de Regió7 sobre l'innovador sorteig. Regió7, 14/06/2017.

Ren, C., y Blichfeldt, B. S. (2011). One clear image? Challenging simplicity in place branding. Scandinavian Journal of Hospitality and Tourism, 11 (4), 416-434. doi:10.1080/15022250.2011.598753

Rius-Ulldemolins, J. Flor Moreno, V. (2017). The dark side of cultural policy: Economic and political instrumentalisation, white elephants, and corruption in Valencian cultural institutions. International Journal of Cultural Policy, 1-16. doi:10.1080/10286632.2017.1296434

Rius-Ulldemolins, J., y Rubio, A. (2013). The governance of national cultural organisations: Comparative study of performance contracts with the main cultural organisations in England, France and Catalonia. International Journal of Cultural Policy, 19 (2), 249-269.

Rius-Ulldemolins, J., y Zarlenga, M. (2014). Industrias, distritos, instituciones y escenas. tipología de clústeres culturales en Barcelona. Revista Española de Sociología, 21, 47-68.

Roggeband, C. (2012). Shifting policy responses to domestic violence in the Netherlands and Spain (1980-2009). Violence Against Women, 18 (7), 784-806. doi:10.1177/1077801212455359

Santamarina, B. (2008). Moros y cristianos. de la batalla festiva a la discursiva. Gazeta de Antropología, 24 (1), 1-10.

Shils, E. (2006). Tradition. University of Chicago Press: Chicago.

Sola, J., y Rendueles, C. (2017). Podemos, the upheaval of Spanish politics and the challenge 
Cultura festiva, política local y hegemonía social: Comparativa de los casos de los Moros i Cristians (Alcoi)...

of populism. Journal of Contemporary European Studies, 1-18. doi:10.1080/14782804.2017.13 04899

Sztompka, P. (1993). The sociology of social change. Hoboken, NJ: Wiley-Blackwell.

Thiesse, A. (1999). La création des identités nationales. Europe, $18{ }^{\circ}-20{ }^{\circ}$ siècle. Editions du Seuil: Paris.

UNESCO. (2008). Intangible heritage. Patum of Berga. Paris: UNESCO.

UNESCO. (2016). Decision of the intergovernmental committee: 11.COM 10.B.30. Paris: UNESCO.

Velasco, H., Cruces, F. et al. Díaz de Rada, A. (1996).

Fiestas de todos, fiestas para todos. Revista de Antropología, 11 (123), 144.

\section{NOTAS BIOGRÁFICAS}

Verónica Gisbert Gracia candidata al doctorado y profesora asociada del Departamento de Sociología y Antropología Social de la Universidad de Valencia. Licenciada en Criminología y Máster Erasmus GEMMA Mundus en Estudios de las Mujeres y de Género. Sus investigaciones se enfocan a la temática de género y política, derechos humanos y procesos de conflicto. Es autora de varios trabajos sobre el movimiento feminista y las festividades tradicionales.

Joaquim Rius-Ulldemolins, doctor en Sociología por la Universidad Autónoma de Barcelona y la École des Hautes Études en Sciences Sociales. Actualmente, es profesor ayudante doctor en el Departamento de Sociología y Antropología Social de la Universidad de Valencia. Es autor de varios libros y artículos sobre la sociología de la cultura y la política cultural en revistas nacionales e internacionales como International Journal of Cultural Policy, Urban Studies, European Urban and Regional Studies, $R E I S, R I S$, entre otras.

Gil-Manuel Hernàndez, historiador y sociólogo, doctor en Geografía e Historia. Actualmente es profesor titular del Departamento de Sociología y Antropología Social de la Universidad de Valencia. Es autor de varios libros, artículos e investigaciones sobre las manifestaciones festivas y rituales la cultura popular, el patrimonio cultural las fiestas y los procesos de globalización. También se ha ocupado del estudio de políticas culturales e identidades colectivas. 